\title{
Source Localization Probability Maps for Uncertainty Quantification in Electrocardiographic Imaging
}

\author{
Jessie France, and Chris R. Johnson
}

\begin{abstract}
This study aimed to develop a new probabilistic visualization analysis to study source localization uncertainty in electrocardiographic imaging (ECGI). Using Monte Carlo error propagation, we developed probability maps that illustrate uncertainty in source localization compared to the ground truth source location. We used these probability maps to quantify the impact of noise amplitude and iterative Krylov regularization on source localization. Artificial Gaussian white noise was added to the body surface potentials between (0.5\% and $9 \%$ of their amplitudes) to simulate noisy observations. We solved the inverse problem to recover heart surface potentials using the conjugate gradient least squares (CGLS) and preconditioned CGLS (PCGLS) algorithms with the Laplacian over the heart surface as a right preconditioner. We forward propagated these inverse solutions, and performed 200 CGLS and PCGLS Monte Carlo inversions per noise level. For each sample, we recorded the top $1 \%$ of lowest potential locations, and normalized across all samples to form empirical probability maps for source localization. Increasing the noise amplitude increased both the uncertainty and inaccuracy for source localization, with PCGLS outperforming CGLS across all noise amplitudes. We conclude that the concept of a source localization probability map may be useful clinically in identifying origins of arrhythmia in cardiac tissue.
\end{abstract}

Keywords - electrocardiographic imaging (ECGI), Krylov inversion, Monte Carlo sampling, uncertainty quantification

\section{Introduction}

Cardiac ablation is an interventional procedure in which a clinician internally locates and destroys regions of cardiac tissue responsible for spontaneous pathological heart beats or reentrant wave activity. However, this invasive therapy can last many hours, and abnormal heart rhythms, or arrhythmias, frequently re-occur following the procedure $[1,2]$. One noninvasive approach to reducing proce-

France, and Johnson are with the Scientific Computing and Imaging Institute, University of Utah. Email: \{jfrance, crj\}@sci.utah.edu. This project was supported by grants from the NIH National Institute of General Medical Sciences (P41 GM103545-18) from the National Institutes of Health. dure times and the risk of recurrence is known as electrocardiographic imaging (ECGI). ECGI uses a patient's CT and MRI images to approximate a computational geometry, and combines this with ECG recordings into a mathematical model that predicts the origin of an arrhythmia.

This inverse problem of electrocardiography may one day become a standard in ablation procedures, allowing clinicians to more quickly and accurately locate pathological heart tissue, since the ECGI prediction for the source of an arrhythmia essentially tells a clinician ahead of time where to ablate [3]. ECGI involves solving Laplace's equation $[4,5]$ to obtain the system

$$
A \mathbf{h}=\mathbf{y}
$$

where $A \in \mathbb{R}^{m \times n}, \mathbf{h} \in \mathbb{R}^{n}$, and $\mathbf{y} \in \mathbb{R}^{m}$. The transfer matrix $A$ relates the heart surface voltages $\mathbf{h}$ to the true body surface voltages $\mathbf{y}^{*}$. However, the observed body surface ECG recordings $\mathbf{y}$ are a combination of the true signal $\mathbf{y}^{*}$ and noise $\mathbf{e}$. In this study, Equation 1 is an underdetermined system (with $m=589$ and $n=2206$ ), and $A$ is severely ill-conditioned. Consequently, solutions to Equation 1 are both ill-posed and non-unique, making the inversion highly sensitive to sources of error in a limited number of torso measurements. Unfortunately bioelectric simulations are sensitive to sources of error [6-9], and there is a lack of quantitative understanding on how noise errors and regularization impact source localization. In this study, we develop probability maps to visualize the impact of noise errors and iterative regularization on the inversion and source localization.

\section{Methods}

In Equation 1, we added artificial, identical and independently distributed Gaussian white noise e with zero mean to the set of true simulated body surface recordings $\mathbf{y}^{*}, e \sim$ $\mathcal{N}(\mathbf{0}, \sigma I)$, where $\sigma$ is the standard deviation of the noise, and $I$ is the identity matrix. Noise was added such that $p=\frac{100\|\mathbf{e}\|_{2}}{\left\|\mathbf{t}^{*}\right\|_{2}}$, where $p$ represents the percent added noise. In this study, we varied $p$ from $0.5 \%$ to $9 \%$. We obtained a solution to Equation 1 using the truncated conjugate gradient least squares method (CGLS) [10-12], with the Morozov discrepancy principle as a stopping criteria [13]. This method applies a series of matrix-vector multiplications 
such that the $k^{\text {th }}$ CGLS solution lies in the Krylov subspace spanned by $\mathbf{h}_{\mathbf{k}} \in\left\{A^{T} \mathbf{y}, \ldots,\left(A^{T} A\right)^{k-1} A^{T} \mathbf{y}\right\}$ [10]. This form of regularization causes $\mathbf{h}$ to converge in the directions of the largest singular values of $A$ [10]. In this study, we also solved Equation 1 using the right preconditioned CGLS (PCGLS) algorithm, using the Laplacian operator $L$ over the heart surface as the right preconditioner. The matrix $L$ was formed as described in [14]. Because $L$ has a non-trivial null-space $W$, the PCGLS method requires formation of the A-weighted psuedo-inverse of $L$ [10], $L^{\#}=\left(I-W(A W)^{\dagger} A\right) L^{\dagger}$, where $I$ is the identity matrix. With $\bar{A}=A L^{\#}$ and $\overline{\mathbf{y}}=\mathbf{y}-A W(A W)^{\dagger} \mathbf{y}$, we solved $\bar{A} \mathbf{z}=\overline{\mathbf{y}}$ for $\mathbf{z}_{\mathrm{k}}$ using the CGLS routine, and obtained the PCGLS solution with $\mathbf{h}_{\mathbf{k}}=L^{\#} \mathbf{z}_{\mathbf{k}}+W(A W)^{\dagger} \mathbf{y}$ [10].

Once we obtained an initial solution $\mathbf{h}_{\mathbf{k}}$ using CGLS or PCGLS, we forward-propagated the solution to form an assumed noise-free right hand side, $\tilde{\mathbf{y}}=A \mathbf{h}_{\mathbf{k}}$ to perform Monte Carlo error analysis as described in [11]. In obtaining a Monte Carlo solution, we sampled a noisy solution $\mathbf{y}_{\mathbf{s}} \sim \mathcal{N}(\tilde{\mathbf{y}}, \sigma \mathbf{I})$, and used the CGLS or PCGLS routines to form an iterative solution $\mathbf{h}_{\mathbf{k}, \mathbf{s}}$ as we did originally in solving Equation 1. We obtained an ensemble of 200 Monte Carlo samples per simulation. To form a probability map, we located the top $1 \%$ of lowest voltages (with the lowest voltage denoting the source), and averaged these locations over the 200 samples to form a probabilistic representation for source localization.

\section{Results}

Figure 1 shows the ground truth heart voltages, the original CGLS and PCGLS solutions, and corresponding Monte Carlo samples for the two algorithms with the noise percent $p$ at $3 \%$. Red dots mark the location of the absolute minimum voltage relative to the ground truth data (the true source origin, which is at the same position on all plots) and white dots mark the estimated locations for each of the estimates. While the CGLS solution and corresponding sample show jagged contour lines, the PCGLS solution and corresponding sample maintain smooth contour lines around the area of the estimated source location. Despite these differences in contour lines representing potential gradients, each of the inverse solutions and their corresponding samples maintain estimated source locations that are within $6 \mathrm{~mm}$ of the ground truth source location. However, to better understand similarities and differences between the CGLS and PCGLS estimations, it is necessary to look at the individual Krylov vectors that make up each solution.
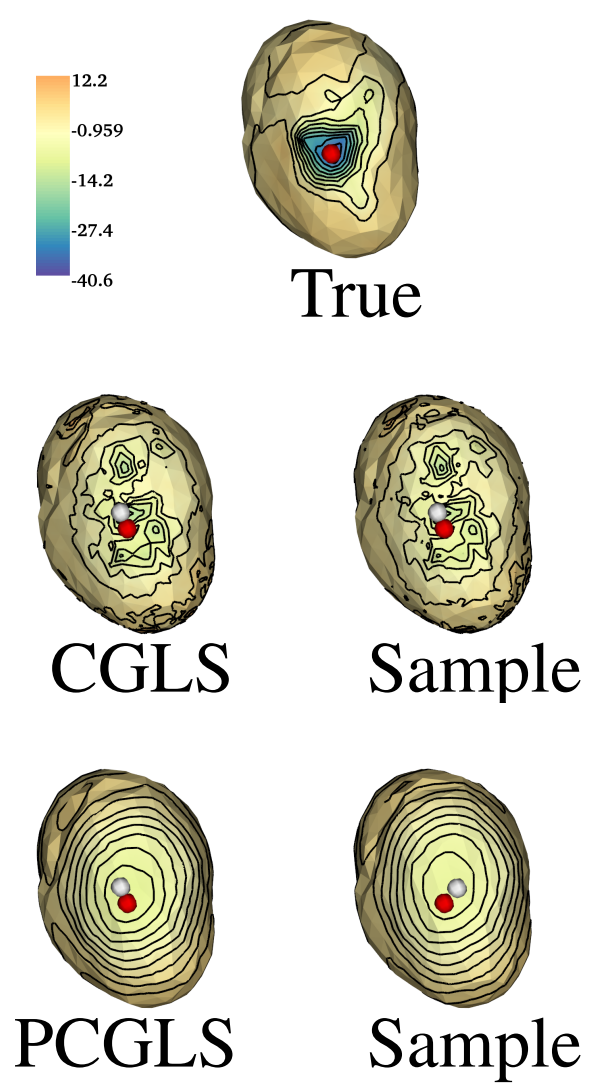

Figure 1. The ground truth solution shows a pronounced minimum marked as a red dot. The initial CGLS solution and Monte Carlo sample show less pronounced minimums (white dots) with non-smooth contour lines. In contrast, the PCGLS solution and its corresponding sample show very smooth contour lines emanating from the source.

Figure 2 shows a selection of Krylov vectors obtained in forming the CGLS and PCGLS solutions in Figure 1. The top row shows the Krylov vectors corresponding to the CGLS solution, and the bottom row shows the Krylov vectors corresponding to the PCGLS solution. Laplacian preconditioning helps guide the solution iterates through a Krylov subspace with smoother contour lines compared to the CGLS solution. These differences in Krylov regularization affect uncertainty in responses to noise errors.

For example, Figure 3 shows probability maps for the CGLS (top row) and PCGLS (bottom row) algorithms in response to ECG noise amplitude relative to the ground truth source location (red dot). Darker green areas indicated regions of higher source localization probability compared with lighter green ares. While the CGLS probability maps tended to disperse into several high probability regions, the PCGLS probability maps aggregated around a single region. At $0.5 \%$, both algorithms produced high 

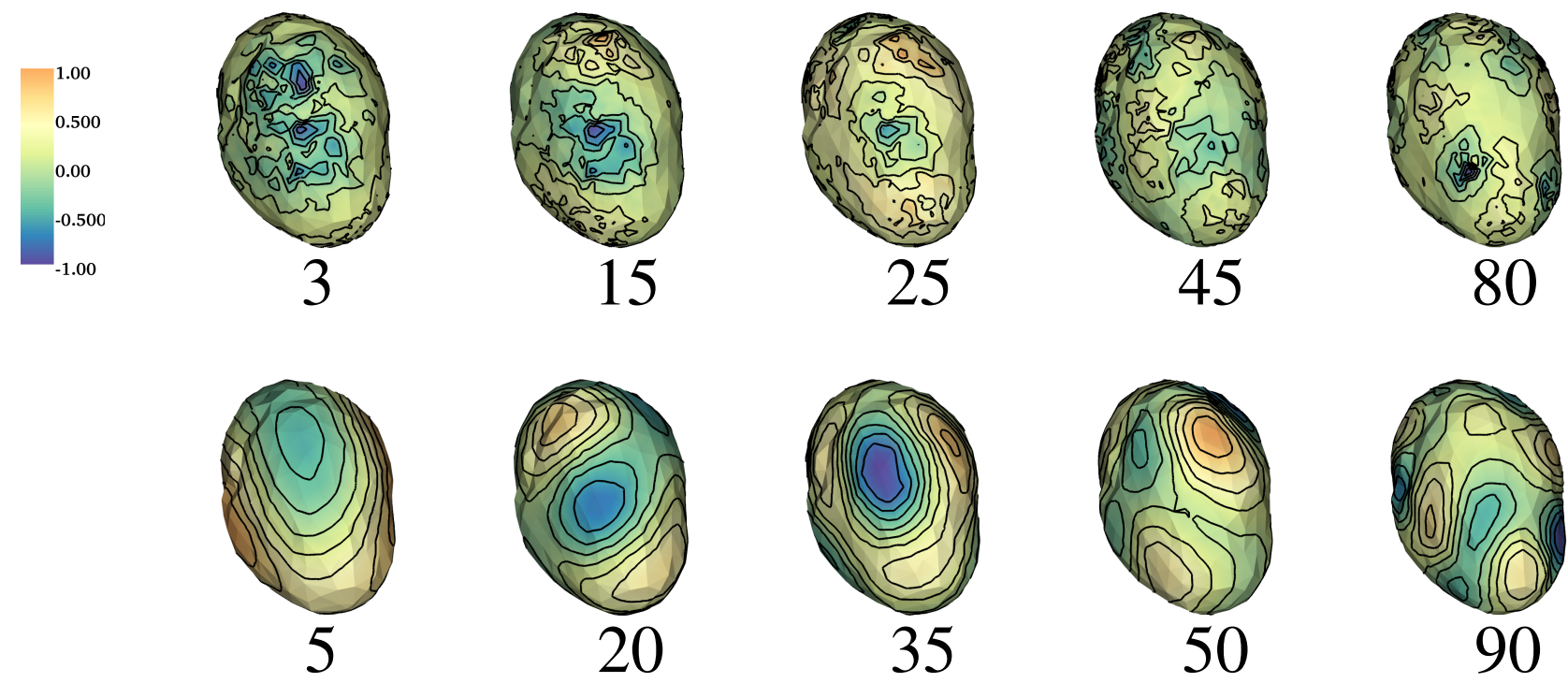

Figure 2. Selection of Krylov vector iterates for CGLS algorithm (top row) and PCGLS algorithm (bottom row). All vectors were normalized to the scale $(-1,1)$.
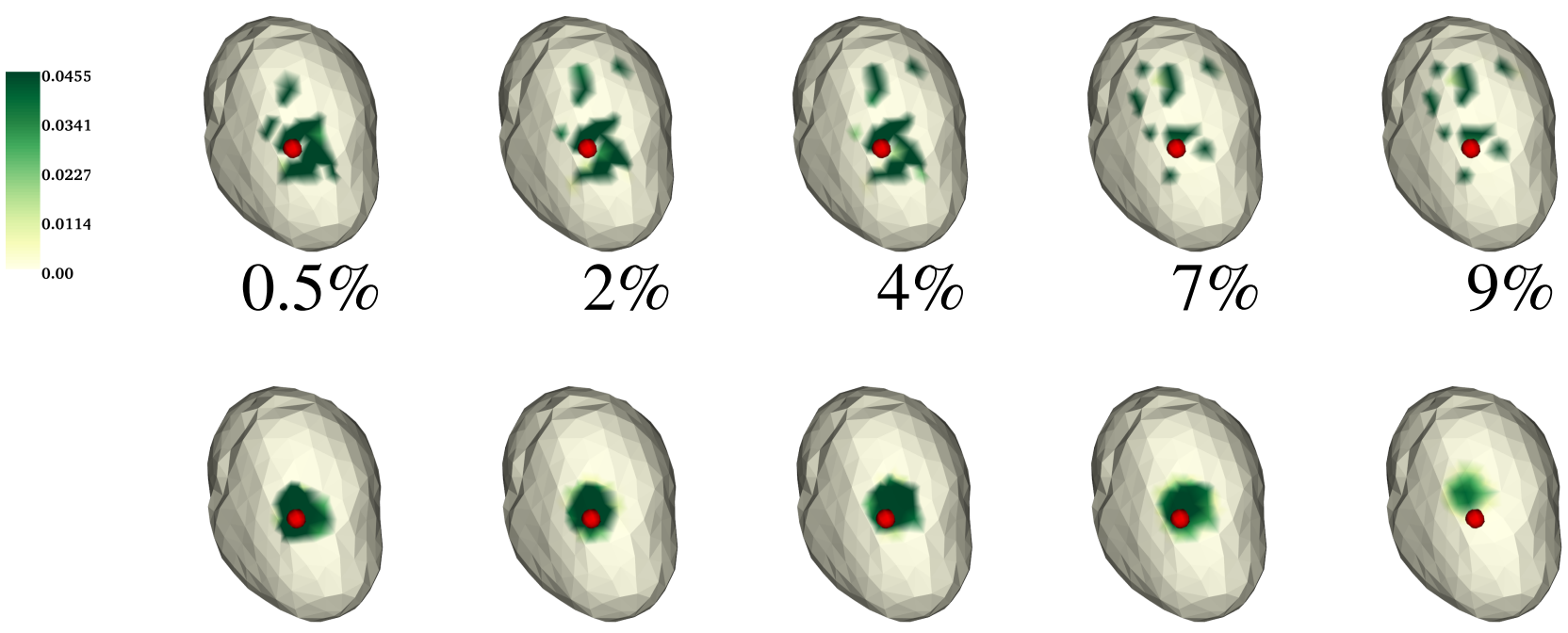

Figure 3. Probability maps for source localization illustrate uncertainty as a function of noise in ECG observations for the CGLS inversion (top row) and PCGLS inversion using a Laplacian preconditioner (bottom row). Dots mark the ground truth source location. 
probability density around the true source. However, at around $7.0 \%$ noise, the high probability region for the CGLS probability maps tended to disperse. In contrast, the PCGLS algorithm maintained a high probability density around the ground truth source. At around 9.0\% noise, the PCGLS algorithm showed a false probability region away from the true source.

While the PCGLS algorithm may help improve source localization accuracy, this algorithm requires more matrixvector multiplications than the CGLS algorithm. Figure 4 shows the absolute error histories (with absolute error defined as $\frac{\left\|\mathbf{h}^{*}-\mathbf{h}_{\mathbf{k}}\right\|_{2}}{\left\|\mathbf{h}^{*}\right\|_{2}}$ relative to the true solution $\left.\mathbf{h}^{*}\right)$ for the CGLS and PCLGS algorithms, and illustrates a trade-off between computational cost and accuracy for these two algorithms. While the PCGLS algorithm tended to converge in a Krylov subspace with less error than the CGLS algorithm, it requires more iterations to reach termination with the Morozov discrepancy principle. In this example with $p=3 \%$, the CGLS algorithm terminated at $k=16$ (with the best possible solution at $k=25$ ), and the PCGLS algorithm terminated at $k=20$ (with the best possible solution at $k=35)$.

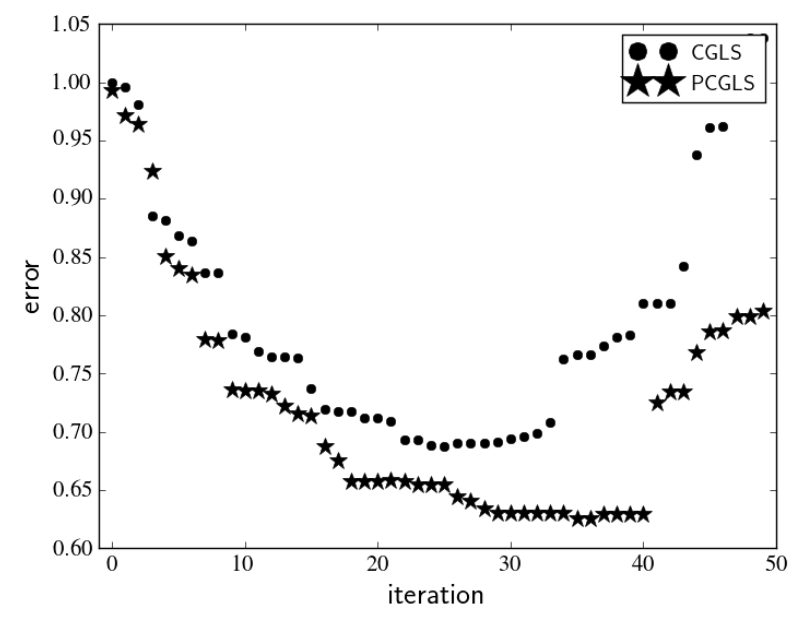

Figure 4. Absolute error histories for CGLS and PCGLS.

\section{Conclusion}

We conclude that the concept of a source localization probability map may be useful clinically in identifying origins of arrhythmia in cardiac tissue. In this study, probability maps illustrated that increasing the noise amplitude increased both the uncertainty and inaccuracy for source localization, with PCGLS outperforming CGLS across all noise amplitudes. Future studies will develop confidence intervals to complement the probability maps, and also explore other sources of uncertainty in electrocardiographic imaging (ECGI).

\section{Acknowledgments}

This project was supported by a grant from the NIH National Institute of General Medical Sciences (P41 GM103545-18). The authors would like to give a special thanks to Professor MacLeod for generously sharing data.

\section{References}

[1] Arya A, et al. Long-term results and the predictors of outcome of catheter ablation of atrial fibrillation using steerable sheath catheter navigation after single procedure in 674 patients. Eurosp 2010;2:173-180.

[2] O'Donnell D, Furniss SS, Dunuwille A, Bourke JP. Delayed cure rate despite early recurrence after pulmonary vein isolation for atrial fibrillation. Am Jour of Cardiol 2003;91:8385 .

[3] Rudy Y. Noninvasive electrocardiographic imaging of arrhythmogenic substrates in humans. Circ Res 2013; 112:863-874.

[4] Wang D, Kirby R, Johnson C. Resolution strategies for the finite element based solution of the electrocardiographic inverse problem. IEEE Transactions on Biomedical Engineering 2011;57(2):220-237.

[5] Wang D, Kirby R, Johnson C. Finite-element-based discretization and regularization strategies for 3-d inverse electrocardiography. IEEE Transactions on Biomedical Engineering 2011;58(6):1827-1838.

[6] Burton B, et al. Uncertainty visualization in forward and inverse cardiac models. In Computing in Cardiology Conference. IEEE Press, 2013; 57-60.

[7] Erem B, van Dam P, Brooks D. Identifying model innaccuracies and solution uncertainties in noninvasive activationbased imaging of cardiac excitation using convex relaxation. IEEE Transactions on Medical Imaging 2014; 33:902-912.

[8] Rosen P, Burton B, Potter K, Johnson C. muView: A visual analysis system for exploring uncertainty in myocardial ischemia simulations. Visualization in Medicine and Life Sciences III 2014;49-69.

[9] France J, Gur Y, Kirby RM, Johnson C. A Bayesian approach to quantifying uncertainty in Tikhonov solutions for the inverse problem of electrocardiography. In Computing in Cardiology. 2014; 529-532.

[10] Hansen P. Discrete Inverse Problems Insight and Algorithms. Philadelphia: Society for Industrial and Applied Mathematics, 2010.

[11] Aster R, Borchers B, Thurber C. Parameter Estimation and Inverse Problems. 2nd edition edition. Boston: Elsevier Incorporated, 2013.

[12] Calvetti D. Preconditioned iterative methods for linear discrete ill-posed problems from a Bayesian inversion perspective. Journal of Computational and Applied Mathematics 2007;198:378-395.

[13] Morozov V. Methods for solving incorrectly posed problems. New York: Springer-Verlag, 1984.

[14] Huiskamp G, van Oosterom A. The depolarization sequence of the human heart surface computed from measured body surface potentials. IEEE Transactions on Biomedical Engineering 1988;35:1047-1058. 